\title{
Size variation of polyaniline nanoparticles dispersed in polyvinyl alcohol matrix
}

\author{
J BHADRA and D SARKAR* \\ Department of Physics, Gauhati University, Guwahati 781 014, India
}

MS received 8 April 2009; revised 19 April 2010

\begin{abstract}
We report the preparation of polyaniline (PANI) nanoparticles dispersed in polyvinyl alcohol (PVA) matrix. From SEM picture it is seen that the particle sizes vary from 100-20 $\mathrm{nm}$. Also with increase in PVA content the stability of dispersion is found to increase. Apart from SEM, spin cast films of PANI in PVA are also characterized through XRD and FTIR. XRD shows increase in crystallinity with PVA content and FTIR gives evidence of crosslinking between PANI and PVA molecules. In plane electrical conductivity (in the range of $10^{2} \mathrm{Scm}^{-1}$ ) and the exponent of nonlinear $I-V$ are found to decrease with increase of PVA content. There is a good correlation between SEM, XRD, FTIR and electrical properties.
\end{abstract}

Keywords. Polymers; nanostructures; composite materials; electrical conductivity.

\section{Introduction}

Since its discovery, polyaniline (PANI) has attracted considerable attention because of its unique electrical and optical properties as well as its ease of preparation and most excellent environment stability among many other conducting polymers (Pei et al 1991; Heeger 2001; Pinto et al 2003; Raghavendra et al 2003; Gaikwad et al 2006). It has numerous applications in electronics, energy storage, anticorrosion coatings, catalyst, chemical sensing etc (Xia and Qi 2001). One of the key problems related to the potential application of PANI is its difficulty in processability, either from melt or from solution. With a rigid $\pi$-conjugated backbone molecular structure, PANI decomposes without melting when heated hindering processability from melt, also it is not soluble in common solvents, so that it cannot be processed from solution either. To overcome this disadvantage (i.e. to increase its processability), various techniques have been adopted. Some of them are substitution in the aniline monomer prior to polymerization, copolymerization with any other suitable polymers, making blends or composites with other conventional synthetic polymers, modifying the preformed Langmuir-Blodgett film (Sarkar et al 1993) and preparation of dispersed colloidal particles (Armes et al 1990; Han et al 2002), and many other variety of techniques. PANI dispersion in synthetic high polymer matrix can be obtained by protecting the aggregate formation using steric stabilizers such as polystyrene sulphonic acid, polyvinyl alcohol and polyethylene oxide etc

*Author for correspondence (sarkardeepali@gmail.com)
(Banerjee and Mandal 1995; Eisazadeh et al 1995; Pinto et al 2003). The particle sizes in these dispersions are in micrometer to nanometer range. There are numerous works on PANI nanocomposites with inorganic hybrids, polymer emulsions in synthetic high polymer, surfactant or micellar medium (Eisazadeh et al 1995; Pethkar et al 1999; Kim et al 2000; Su and Kuramoto 2000; Paul et al 2007). These kinds of PANI dispersion can be used as transparent coating for EMI shielding antistatic coating, display devices and electrodes, corrosion protection for metals etc. In these applications PANI grains are of nanometer dimension and this type of nano dispersion is believed to be superior to its bulk (micro or higher sized grains) counterpart (Han et al 2002; Wang et al 2008) in many aspects.

There have been enumerable number of works on PANI using PVA as steric stabilizer (Trivedi and Dhawan 1991; Quadrant et al 1995; Sarkar et al 2005). Most of these works were carried out either in organic acid medium or functionalized acid medium developed in bulk phase. It is not clear how to regulate the shape and size of these PANI particles in such colloidal dispersions. The physical and chemical properties of PANI, such as electrical conductivity, solubility and percolation thresholds in composites can vary significantly with size and morphology of PANI particles (Zheng et al 1997). So far, to the best of our knowledge, there are no significant studies in the direction of synthesis of stable nanometer-sized dispersion of PANI in PVA from aqueous inorganic acid $(\mathrm{HCl})$. So we thought it worthwhile to carry out extensive study in this particular direction. In this article, we focussed on the preparation of PANI nanoparticles of various sizes in PVA matrix by controlling PANI-PVA molar ratio. This 
is because PANI nanoparticles have special electrical and optical properties different from bulk PANI and also with decrease in particle size. PANI dispersion medium becomes more stable and uniform which is helpful to produce uniform, transparent film. To be particular, we report synthesis of polyaniline nano in PVA matrix for three different molar ratios of PANI to PVA. The films are characterized by FESEM, XRD, and FTIR for morphology, grain size, crystallinity and cross-linking assignments, respectively. We have also studied electrical conductivity of these films and sought for possible correlation with various characterization results.

\section{Experimental}

\subsection{Materials}

All the chemicals and reagents used in the present study were obtained from Emerck (Germany) and Sigma Chemical Company and are of very high purity (99.9\%). However, aniline was purified by repeated distillation under vacuum prior to use.

\subsection{Sample preparation}

Polyaniline nanodispersion in PVA was achieved by dispersion polymerization. This was achieved by the conventional technique for preparation of PANI. To the solution of PVA and aniline (in molar ratios of 5, 7 and 9) in aqueous HCL $(0 \cdot 1 \mathrm{M})$, ammonium persulphate $(0 \cdot 1 \mathrm{M})$ was added drop wise. The bath temperature was maintained at $-5^{\circ} \mathrm{C}$. Within few hours the mixture turned into a bluish green homogeneous solution without any trace of sediment. The films were spin cast on various substrates from this solution, dried and washed with methanol and deionized water to remove any oligomer or unreacted oxidant.

\subsection{Characterization}

The particle size and morphology of films spin cast on glass substrate were studied by FESEM images. These were recorded by scanning electron microscope (JSM$6700 \mathrm{~F}$, JEOL, Japan). The particle size range vs frequencies of occurrence of histograms have been obtained from these SEM pictures. This was done by cropping the SEM image by 'Image' software for 500 particles noting their size. The data were then transferred to 'Origin 7.0 ' to give the needed histogram. X-ray diffraction data for crystallinity study of the films are taken by Scifert XRD $3000 \mathrm{P}$ diffractometer with $\mathrm{CuK}_{\alpha}$ radiation (1.5418 $\AA$ ). FTIR spectra of these films were recorded to assign the type of cross linking between PANI and PVA. These were recorded by Perkin Elmer photometer (Model RV5).

\subsection{Electrical properties}

A standard four-probe method was used for the electrical conductivity measurements. Films cast on ITO coated glass were used to measure the $I-V$ characteristics in the direction perpendicular to the plane of the film. For these measurements use was made of Keithley constant current source (model-220), Keithley CV meter (model 595) and a Hewlett Packard multimeter (E2373A).

\section{Results and discussion}

\subsection{Stability of dispersion}

The stability of the polyaniline dispersion in PVA is seen to increase with PVA content and is found to be highest in the film for $9 \mathrm{M} / \mathrm{M}$ concentration. This particular solution appears clear and stays for more than fifteen days without any sedimentation or phase segregation. It may be worthwhile to mention that the nanodispersion is not very stable for PVA concentration of less than $5 \mathrm{M} / \mathrm{M}$. In one of our recent work (Bhadra and Sarkar 2009), we have shown that the dispersion is most stable for PVA concentration of $11 \mathrm{M} / \mathrm{M}$.

\subsection{Particle size and morphology of polyaniline}

The FESEM pictures of the PANI-PVA films are shown in figure $1(\mathrm{a}-\mathrm{c})$ for different PVA contents of 5, 7 and $9 \mathrm{M} / \mathrm{M}$, respectively. The homogeneity of film surface is seen to increase with increase in PVA content. The particle size frequency histogram obtained from these SEM pictures are also shown in the same figure, which show maximum frequency of particle sizes, $100 \mathrm{~nm}, 55 \mathrm{~nm}$ and $24 \mathrm{~nm}$, respectively. However, for the $9 \mathrm{M} / \mathrm{M}$ film the granules are more or less of same size. This clearly shows that particle size agglomeration decreases with increase in PVA content. The average particle size decreases from 100 to $20 \mathrm{~nm}$ (table 1). Another interesting feature is also observed here, viz. with increasing PVA content, the particle size attains near spherical (for $5 \mathrm{M} / \mathrm{M}$ ) to spherical (for $9 \mathrm{M} / \mathrm{M}$ ). Further in this particular film, the spherical granules show some trend to align along a particular direction. For lower PVA content the polyaniline nanoparticles formed are larger in size, possibly because large number of particles aggregated (Xia and Wang 2002) to form larger sized particles. Increase of PVA facilitates regular shape and alignment of the nanoparticles as the interaction with increasing number of PVA molecules (capping) hinders aggregate formation and inhomogeneity. It indicates increase of order with the increase of PVA content. This fact is also strengthened by our recent paper where we have shown that increase of PVA concentration to $11 \mathrm{M} / \mathrm{M}$ facilitates nanorod formation (Bhadra and Sarkar 2009). 

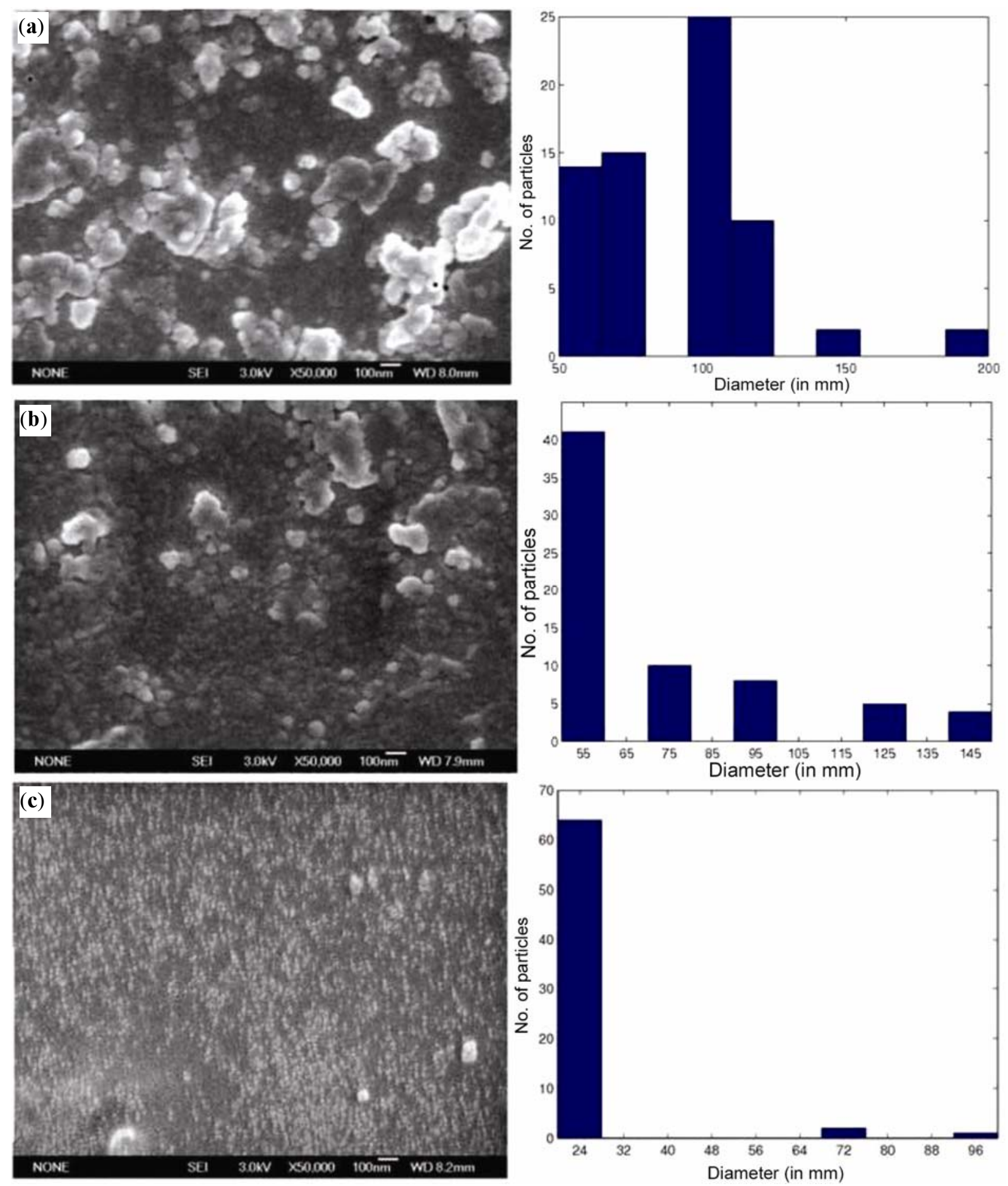

Figure 1. FESEM and frequency histogram pictures for films of PANI-PVA: (a) $1: 5$, (b) $1: 7$ and (c) $1: 9$.

\subsection{Crystallinity of composites}

X-ray diffraction pattern for the PVA-PANI films are shown in figure 2(b-d) for different PVA contents. We also show XRD of a pure polyaniline film (figure 2a) which shows characteristic amorphous peak of PANI at $2 \theta$ values of 11.20 and 19.8 (Jozefowicz et al 1989; Yang and Wan 2002; Lida and Frederise 2003; Seoudi et al 2008) corresponding to (011) and (200) planes, respectively. Also there is an amorphous peak of PVA at a $2 \theta$ value of $19.54^{\circ}$. We observe that with increase of PVA content this peak diminishes with generation of small hump near the peak corresponding to (200) plane (Yang and Wan 2002). Also, at a $2 \theta$ value of $33 \cdot 2^{\circ}$ [correspond- 
ing to (104) plane (Huang et al 2008)], an extra peak starts to appear for $7 \mathrm{M} / \mathrm{M}$ film and this is more distinct for $9 \mathrm{M} / \mathrm{M}$ ratio. These results indicate that with increase of PVA, more crystallinity is induced in the films. This is in conformity with the FESEM pictures which show more regularity in alignment for higher PVA ratio.

\subsection{FTIR}

In figure 3 we show FTIR spectra of free standing PANIPVA films for PVA contents of 5, 7 and $9 \mathrm{M} / \mathrm{M}$. We also show FTIR spectra for pure PANI and PVA for comparison. Pure PANI shows strong IR absorption at a wave number of $3430 \mathrm{~cm}^{-1}$ and that of pure PVA at $3460 \mathrm{~cm}^{-1}$. These are characteristic absorption for $\mathrm{C}-\mathrm{H}$ stretch and O-H stretch, respectively (Lida and Frederise 1993). The composite films show strong absorption around this range

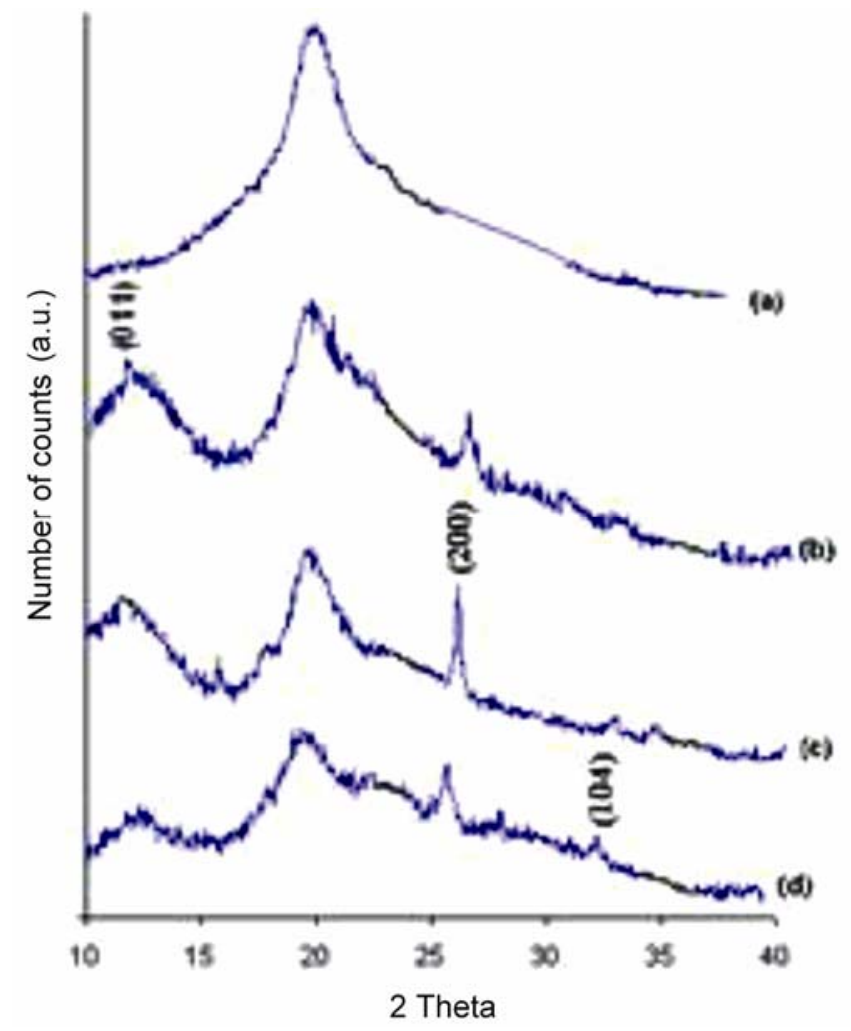

Figure 2. X-ray diffraction spectra of PANI-PVA films: (a) $1: 0$, (b) $1: 5$, (c) $1: 7$ and (d) $1: 9$.

Table 1. Grain size, conductivity and slope of PANI particles of various films.

\begin{tabular}{lccc}
\hline $\begin{array}{l}\text { PANI: } \\
\text { PVA(M/M) }\end{array}$ & $\begin{array}{c}\text { Grain size } \\
(\mathrm{nm})\end{array}$ & Slope $(n)$ & $\begin{array}{c}\text { Conductivity } \\
(\mathrm{S} / \mathrm{m})\end{array}$ \\
\hline $1: 5$ & 100 & $0 \cdot 20$ & 125 \\
$1: 7$ & 50 & $0 \cdot 19$ & 96 \\
$1: 9$ & 20 & $0 \cdot 15$ & 84 \\
\hline
\end{tabular}

with split in absorption peaks and the position lie between 3431 and $3146 \mathrm{~cm}^{-1}$. This shows clear indication of some sort of cross-linking to disturb the perfect $\mathrm{C}-\mathrm{H}$ and $\mathrm{O}-\mathrm{H}$ stretching of pure materials. The figure also shows bands at 1650 and $1435 \mathrm{~cm}^{-1}$ which are characteristic bands of the $\mathrm{C}-\mathrm{C}$ stretching of quinoid and benzenoid rings (Lida and Frederise 1993) and $\mathrm{C}-\mathrm{N}$ stretching modes at 1327 and $1219 \mathrm{~cm}^{-1}$. The characteristic $\mathrm{C}-\mathrm{C}$ ring stretching peak at $1633 \mathrm{~cm}^{-1}$ (Lida and Frederise 1993) is seen to shift to the higher wavelength side. The shift is maximum for 1:9 ratio. Further in the composite films the absorption band is seen at or around $1401 \mathrm{~cm}^{-1}(\mathrm{C}-\mathrm{O}$ stretch) and $1185 \mathrm{~cm}^{-1}$ (Ph-CH bend) (Lida and Frederise 1993; Yang and Wan 2002; Chen et al 2008), the absorption is much stronger than that of pure samples. All these results indicate very strong cross-linking between the PVA and the PANI molecules in dispersion.

\subsection{Electrical conductivity}

The electrical conductivity of the composite films ranged from $1.25-8.4 \times 10^{2} \mathrm{Sm}^{-1}$. There is a decrease in conductivity value with increase in PVA content. This is justified as there is a decrease in the number of more conducting species, i.e. polyaniline in the composite with higher PVA content. This has been observed in some other polyaniline composites also (Karakisla et al 1996). However, our observation on SEM and XRD data depict

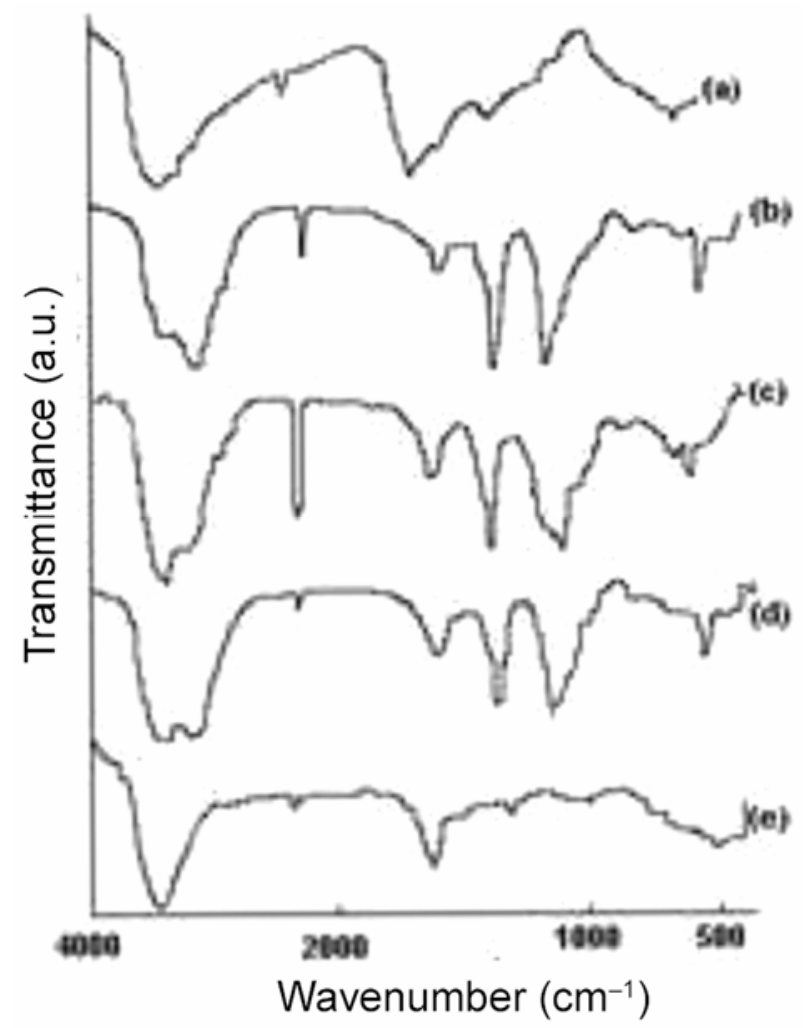

Figure 3. FTIR spectra of PANI, PVA and PANI-PVA films: (a) PANI, (b) $1: 5$, (c) $1: 7$, (d) $1: 9$ and (e) PVA. 
increasing order and more proximity to nanophase with increasing PVA content. This feature is found for many materials in nanophase where it is seen that with decrease in particle size and increase in order, the materials show behaviour very different from that of its bulk phase. Mostly their optical properties enhance at the cost of electrical properties. We show the conductivity values for different PANI-PVA films in table 1 along with the particle size obtained from FESEM results. We have also measured the current-voltage $(I-V)$ characteristics of these films on ITO coated glass slides. These follow nonohmic behaviour showing current value to have power law dependence i.e. $I \propto V^{n}$. The value of $n$ is estimated to be $0 \cdot 20-0 \cdot 15$ as PVA content changed from 5-9 M/M. The $n$ values are also shown in the same table 1 . Nonohmic behaviour of $I-V$ characteristics indicates possibility of these nanocomposite films to have some device applications.

\section{Conclusions}

Nanometer sized PANI particles have been successfully prepared by dispersion polymerization in PVA matrix. The FESEM pictures confirm formation of nanometer sized particles. The aggregation of particles is seen to decrease with increase in PVA. Sizes of nanoparticles also show systematic decrease with the increase in PVA content. In the film with $9 \mathrm{M} / \mathrm{M}$ of PVA content, the nanospheres are almost of equal size and they show alignment along one particular direction. XRD studies reveal the increase of crystallinity with PVA concentration. Possible interaction of PANI-particles with PVA is also indicated from FTIR spectra. The non-ohmic $I-V$ characteristics suggest possible application of these films in the field of device (particularly FET etc) fabrication.

\section{Acknowledgements}

The authors express their deep sense of gratitude to Dr $M$ Ghosh, IACS, Kolkata, for FESEM and XRD measurements and to Dr P K Boruah, Chemistry Department, Gauhati University, Guwahati, for FTIR measurements. Authors acknowledge DST for instrument facilities. One of the authors (JB) acknowledges UGC for providing RFSMS research fellowship.

\section{References}

Armes S P, Aldossi M, Agnew S F and Gottesfeld S 1990 Langmuir 61745
Banerjee P and Mandal B M 1995 Macromolecules 283440

Bhadra J and Sarkar D 2009 Mater. Letts 6369

Chen J, Guo Y, Jiang B and Zang S 2008 Disp. Sci. Technol. 29 97

Eisazadeh H, Spinbs G, Gilmore K J, Hodgson A J and Wallace G 1995 Colloid. Surf. A103 281

Gaikwad P D et al 2006 Bull. Mater. Sci. 29169

Han M G, Cho S K, Oh S G and Im S S 2002 Synth. Met. 126 53

Heegar A J 2001 Curr. Appl. Phys. 1247

Huang H, Feng X and Zhul J 2008 Nanotechnology 19 145607

Jozefowicz M E, Laversanne R, Javadi H H S and Epstein A J 1989 Phys. Rev. B39 17

Karakisla M, Sacale M and Akbulut U 1996 J. Appl. Polym. Sci. 591347

Kim B J, Oh S G, Han M G and Im S S 2000 Langmuir 16 5841

Lida D R and Frederise H P R 1993 Handbook of chemistry and physics (Boca Raton, Florida: CRC Press)

Paul G K, Bhaumik A, Patra A S and Bera S K 2007 Mater. Chem. \& Phys. 106360

Pei Q, Yu G, Zhang C, Yang Y and Heegar A J 1991 Science 2691086

Pethkar S, Patil R C, Kher J A and Vijayamohanan K 1999 Thin Solid Films 349105

Pinto N J, Johnson A T, MacDiarmid A G, Mueller C H, Theofylaktos N, Robinson D C and Miranda F A 2003 Appl. Phys. Lett. 834224

Quadrant O, Steijskal J, Klason C, Kubat J and McQuim D H 1995 J. Phys. Condens. Matter 73287

Raghavendra S C, Khasim Syed, Revanasiddappa M, Ambika Prasad M V N and Kulkarni A B 2003 Bull. Mater. Sci. 26 733

Sarkar A, Ghosh P, Meikap A K, Chattopadhyay S K, Chatterjee S K and Ghosh M 2005 J. Appl. Phys. 97113713

Sarkar D, Misra T N and Paul A 1993 Thin Solid Films 30 255

Seoudi R, Abd El Mongy S and Shabaka A A 2008 Physica B: Condens. Matter 4031781

Sharma Raksha, Malik Rakesh, Lamba Subhalakshmi and Annapoorni S 2008 Bull. Mater. Sci. 31409

Su S J and Kuramoto N 2000 Synth. Met. 114147

Trivedi D C and Dhawan S K 1991 Frontiers of polymer research (eds) P N Prasad and J K Nigam (New York: Plenum) p. 419

Wang X J, Liu J, Feng X, Guo M and Sun D 2008 Mater. Chem. Phys. 112319

Xia H and Qi Wang 2001 J. Nanoparticle Res. 3401

Xia H and Qi Wang 2002 Chem. Mater. 142158

Yang Y and Wan M J 2002 Mater. Chem. 12897

Zheng W, Angelopoulos M, Epstein A J and MacDiarmid A G 1997 Macromolecules 307634

Zheng W Y, Levon K, Laasko J and Osterholm J E 1994 Macromolecules 277754 